\title{
Author Index Vol. 35, 1997
}

\section{$(A)=$ Abstracts}

\section{Achermann, P. 161(A)}

Adler, $\mathrm{L} \cdot 205$

Akopov, G.P. 156 (A), 163 (A),

167(A) Allard, P. 11 Almirall, H. 84 Almkvist, O. 171(A) Alper, K. 156(A) Amore, M. 197 Andelkovic, D. 168(A) Anderer, P. 156 (A), 158 (A) Andreasen,

N.C. 61 Andronova, T. 163(A) Androsiuk, W. 157(A),

159(A), 169(A) Antonijoan, R.M. 158(A) Aoyama, T. 91

Ardizzone, G. 157 (A), 167 (A) Arrigo, A. 157 (A), 167 (A) Arruda, S. 24 Ashkenazi, A. 178 Avakian, G.N. 158(A) Avseenko, N.D. 157(A)

Badalian, O.L. 158(A) Barboni, M.J. 158(A) Basinska, A. 158 (A), 159 (A) Battaglia, M. 164(A) Battegay, R. 162(A) Beranek, J. 158(A) Berardi, D. 197

Bernardo, A. 161 (A) Bischof, R. 162(A) Boeijinga, P. 170(A) Bojovic, L. 168(A) Boni, S. 73,79 Borbély, A.A. 161(A) Brambilla, F. 1 Brandão, M.L. 30 Brichcin, S. 159(A) Bringmann, A. 102 Brüderlin, U. 162(A) Brunello, N. 57 Bucci, P. 161(A) Bulotta, S. 168(A) Burger, R.A. 181

Caetano, D. 24 Caffarra, P. 73, 79 Catapano, F. 166(A) Chaba, P. 158 (A), 159 (A) Chiodera, P. 73, 79 Clincke, G. 160(A) 
Cluydts, R. 115 Coenen, A.M.L. 171(A) Coiro, V. 73, 79 Costa e Silva, J.A. 24 Czobor, P. 159(A)

Dardano, D. 167(A) David, I. 159(A), 160(A) Davis, J.M. 5 Decobert, M. 160(A) De Meirleir, K. 115 Demicheli, F. 164(A) Depoortere, H. 160(A) Dimpfel, W. 132 Dirksen, R. 171(A) Dominguez, N. 158 (A) Dugovic, C. 160(A) Duzyurek, S. 61

Earley, B. 200 Edman, A 160(A) Endo, S. 172(A) Engelsmann, F. 95 Eriksson, A. 11 Ernst, A. 162(A)

Fell, J. 108 Feshchenko, V.A. 211 Finelli, L. 161(A) Fischler, B. 115 Flaum, M. 61 Francon, D. 160(A) Fries, U. 143 Friess, E. 147 Fukue, M. 128 Gabrielli, W.F. 159(A) Galderisi, S. 161 (A), 166 (A) Gamundí, A. 84 Gershovich, Y. 163(A) Giglioli, D. 165(A), 168(A) Gnezditsky, V.V. 156(A), 161(A), 163(A) Gobert, J. 164(A) Goodwin, D.W. 159(A) Goto, T. 91 Grön, G. 147 Gschwandtner, U. 162 (A) Guareschi-Cazzullo, A. 1 Guilpin, C. 221 Gupta, S. 175 
Hara, T. 91 Hemmeter, U. 162(A) Herpers, M. 147 Hirota, T. 46, 172(A) Hode, Y. 170(A) Hoffmann, G. 115 Holsboer-Trachsler, E. 162 (A) Huether, G. 205 Hyoki, K. 169(A)

Ichinowatari, N. 91 Igel, G. 175 Ilic, K. 162(A) Inagaki, T. 187 Irwin, P. 159(A) Ishino, H. 187

Jampala, V.C. 175 Jane, F. 158(A) Jelic, V. 171(A) Jensen, P. 159(A) John, E.R. 156(A) Jongsma, M.L.A. 171(A) Judd, L.L. 57 Julin, P. 171(A)

Kagaya, A. 128 Kajimura, N. 152 Kalinina, T.S. 164(A) Kamath, S.K. 5 Kane, J.M. 64 Kato, M. 152 Kaufman, L. 115 Kay, J. 175 Kinoshita, T. $46,169(A)$,

171(A), 172(A) Kissling, W. 70 Kleinlogel, H. 162(A) Knop, J. 159(A) Kobayashi, S. 187 Kocher, R. 162(A) Kochi, K. 46, 172(A) Koenig, T. 46, 172(A)

Kojima, T. 160(A) Kondakor, I. 46, 172(A) Korepina, O.S. 156(A),

161(A), 163(A) Koukkou, M. 162(A) Kovalzon, V. 163(A) Kraineva, V.A. 164(A) Krijzer, F. 167(A) Kristbjarnarson, H. 163(A) Kristjansson, S. 163(A) 
Kugaya, A. 128 Kuntsevich, M. 163(A) Kunze, K 143

LaNoce, A. 164(A) Langer, S.Z. 57 LeBon, O. 115 Lehmann, D. 46, 161 (A),

169(A), 171(A), 172(A) Leonard, B.E. 200 Limoge, A. 221 Liogier d'Arduy, X. 170(A) Loizzo, A. 164(A) Loizzo, S. 164(A) Lopez, L. 169(A) Luthringer, R. $170(\mathrm{~A})$

Macher, J.-P. 170(A) Maciulis, A. 181 Maj, M. 161(A), 166(A) Malvezzi, L. 73 Mannaerts, H. 51 Marcusson, J. 11 Margineanu, D.-G. 164 (A) Markina, N.V. 158 (A), 164 (A) Markina, N.Y. 157(A) Marra, R. 168(A) Masaki, K. 91 Massana, E. 158(A) Massimilla, S. 165 (A), 168 (A) Matousek, M. 160 (A), 165 (A) Matsubara, K. 187 Matsumoto, H. 157(A),

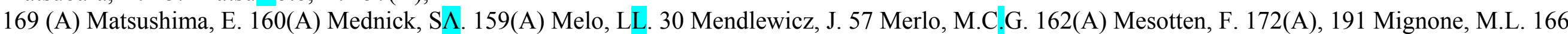
(A) Miller,D. 61 Milovanovic, D. 168(A) Minagawa, H. 128 Miragoli, L. 164(A) Molnar, G. 165(A) Morvan, C, 160(A) Mucci, A. 161(A), 166(A) Müller, H.F. 95 Munjiza, M. 166 (A), 173 (A) Musetti, C. 1

KAIIGEI $\mid$ 
(C) 1997 S. KargerAG, Basel

E-Mail karger@karger.ch Fax + 41613061234 http://www. karger. ch

Nakamura, S. 172(A) Narici, L. 169(A) Narkina, N.V. 166(A) Nerobkova, L.N. 158(A),

164(A), 166(A) Nicolau, M.C. 84 Nievelstein, H. 167(A)

Obradovic, D. 168(A) Odell, J.D. 181 Okuma, T. 152 Okunishi, H. 187 Oyamada, T. 128 Ozun, M. 24

Palazzesi, S. 164(A) Panerai, A.E. 1 Parra, P. 158(A) Pascual-Marqui, R.D. 156(A),

161 (A) Penick, E.C. 159(A) Petrova, E. 156 (A), 167 (A) Pilz, J. 205

Plekhanova, S.A. 163(A) Plioplys, A.V. 16 Plioplys, S. 16 Prasad, A.S. 175 Priano, F. 157 (A), 167 (A) Prichep, L.S. 156(A)

Racagni, G. 57

Radziwon, M. 157 (A), 169 (A) Rammsayer, T.H. 36 Reddy, M.N. 175 Reinsel, R.A. 211 Rial, R.V. 84 Rinaudo, G. 170(A) Rispoli, V. 168(A) Robert, C. 221 
Robitaille,YY. 95 Rocca, G. 165(A), 168(A) Rocha, F.L. 24 Röschke, J. 108 Roselló, C. 84 Rotiroti, D. 168(A) Rupprecht, R. 147 Ruschel, S.I. 24 Rusic, D. 162(A), 168(A) Rybakowski, J.K. 123

Sacerdote, P. 1 Saemundsson, R. 163(A) Saginario, A. 73, 79 Saletu, B. 156 (A), 158 (A) Sandner, G. 30 Sannita, W.G. 157 (A), 165 (A),

167(A), 168(A), 169(A) Sansone, M. 164(A) Scaglioni, A. 73,79 Schellenberg, R. 132 Schober, F. 132 Schulsinger, F. 159(A) Schultz, S. 61 Seifertova, D. 160(A) Semlitsch, H.V. 156(A) Sever, Y. 178 Shapiro, L.E. 5 Sharma, R.P. 5 Shigeta, M. 169(A), 171(A) Shikimi, T. 187 Shimazono, Y. $160(A)$ Shimizu, K. 91 Silva Lippi, J.R. da 24 Skalski, M. 157 (A), 169 (A),

170(A) Sluzewska, A. 123 Sobieska, M. 123 Sobolewski, E. 175 
Soll, E.A. 5 Song, C. 200 Souêtre, E. 67 Soufflet, L. 170(A) Stürenburg, H.J. 143 Sumsky, L.I. 170(A) Sundman, I. 11 Susie,|V. 173(A) Suzuki, H. 172(A) Svadovsky, A.I. 161(A) Szelenberger, W. 157(A),

158(A), 159(A), 169(A),

170(A), 172(A)

Tacchini, C. 1 Takaori, S. 187 Takebayashi, M. 128 Takezaki, E. 128 Ternirbaeva, C. 156(A) Timmerman, H. 167(A) Tiplady, B. 159(A) Tirone, P. 164(A) Todorova, A. 132 Toussaint, M. 170(A) Troncoso, A.C. 30 Tsakalidou, A. 159(A) Tsukahara, Y. 152 Tsukano, K. 128 Tuno, N. 169(A) Tyano, S. 178 Uchitomi, Y. 128 Usui, M. 171(A)

Van den Broeck, W. 160(A) Van den Broek, P.L.C. 171 (A) Vanderspickken, C. 51 VanEgmond, J. 171(A) Van Erp, M.G. $172(A), 191$ 
Van Rijn, CM. 171(A) Van Sweden, B. 51, 172(A),

191 Vasavan Nair, N.P. 95 Verbaten, M.N. 172(A) Veselis, R.A. 211 Volavka, J. 159(A) Volpi, R. 73,79 Voronina, T.A. 158(A),

164(A), 166(A)

Wackermann, J. 46, 158 (A), 165(A), 170(A), 172(A) Wahlund, L.-O. 171(A) Wallin, A. 160(A) Warren, R.P. 181 Warren, W.L. 181 Watanabe, M. 91 Watanabe, M.D. 5 Wedekind, D. 205 Wedekind, W. 132 Weizman, A. 178 Weniger, G. 205 Winblad, B. 171(A)

Yagyu, T. 46, 172(A) Yamadera, H. 152, 172(A) Yamawaki, S. 128 Yeragani, V.K. 175 Yoshimasu, H. 91 Yoshino, A. 91

Zazzeri, N. 197 Zdravkovic-Munjiza, B.

166(A), 173 (A) Zensho, H. 128 Zivanovic, D. 173(A)

Author Index 
